Case Report

\title{
Protracted Hypocalcemia following 3.5 Parathyroidectomy in a Kidney Pancreas Recipient with a History of Robotic-Assisted Roux-en-Y Gastric Bypass
}

\author{
Hugo Bonatti $\mathbb{D}^{1,2}$ Naureen Iqbal, ${ }^{1,3}$ Catherine Kling, \\ Willie Melvin, ${ }^{1,5}$ and James Broome ${ }^{1,6}$ \\ ${ }^{1}$ Department of Surgery, Vanderbilt University Medical Center, Nashville, TN, USA \\ ${ }^{2}$ Meritus Surgical Specialists, Hagerstown, MD, USA \\ ${ }^{3}$ Geisinger, Wilkes-Barre, PA, USA \\ ${ }^{4}$ Surgery, University of Washington, Seattle, WA, USA \\ ${ }^{5}$ StoneCrest Medical Center Clinic, Smyrna, TN, USA \\ ${ }^{6}$ St. Thomas Endocrine Surgical Specialists, Nashville, TN, USA
}

Correspondence should be addressed to Hugo Bonatti; hugo.bonatti@dr.com

Received 30 March 2018; Accepted 12 June 2018; Published 24 July 2018

Academic Editor: Marian Klinger

Copyright (C) 2018 Hugo Bonatti et al. This is an open access article distributed under the Creative Commons Attribution License, which permits unrestricted use, distribution, and reproduction in any medium, provided the original work is properly cited.

Background. Hypocalcemia is a frequent complication of parathyroidectomy for secondary/tertiary hyperparathyroidism. In patients with a history of prior Roux-en-Y gastric bypass (RYGBP), changes in nutritional absorption make management of hypocalcemia after parathyroidectomy difficult. Case Report. A 41-old-year morbidly obese female with c-peptide negative diabetes mellitus and renal failure had RYGBP. Following significant weight loss she underwent simultaneous pancreas-kidney transplantation. She had excellent transplant graft function but developed tertiary hyperparathyroidism with calciphylaxis. She underwent resection of 3.5 glands leaving a small, physiologic remnant remaining in situ at the left inferior position. She was discharged on postoperative day one in good condition, asymptomatic with serum calcium of $7.6 \mathrm{mg} / \mathrm{dL}$ and intact PTH of $12 \mathrm{pg} / \mathrm{mL}$. The patient had to be readmitted on postoperative day \#14 for severe hypocalcemia of $5.0 \mathrm{mg} / \mathrm{dl}$ and ionized calcium $2.4 \mathrm{mg} / \mathrm{dl}$. She required intravenous calcium infusion to achieve calcium levels of $>6.5 \mathrm{mg} / \mathrm{dl}$. Long-term treatment includes $5 \mathrm{~g}$ of elemental oral calcium TID, vitamin D, and hydrochlorothiazide. She remains in the long term on high-dose medical therapy with normal serum calcium levels and PTH levels around $100 \mathrm{pg} / \mathrm{mL}$. Discussion. Our patient's protracted hypocalcemia originates from a combination of 3.5 gland parathyroidectomy, altered intestinal anatomy post-RYGBP, and potentially her pancreas transplant causing additional metabolic derangement. Alternative bariatric procedures such as sleeve gastrectomy may be more suitable for patients with renal failure or organ transplants in whom adequate absorption of vitamins, minerals, and drugs such as immunosuppressants is essential.

\section{Introduction}

Pancreas transplantation (PTx) has evolved as a reliable therapy for insulin-dependent diabetes mellitus (IDDM) and most commonly it is performed as a simultaneous pancreas-kidney transplant (SPK) in the setting of diabetic nephropathy $[1,2]$. Only a limited number of patients with non-IDDM have thus far undergone PTx if they were able to lose significant weight and still remain insulin-dependent [35]. Obesity has become one of the most common disorders worldwide, and morbid obesity is often a contraindication for kidney and pancreas transplant listing. As nonoperative measures are unsuccessful in the majority of obese individuals, bariatric surgery has gained importance in the therapy of morbid obesity [6]. Currently in the US, the Roux-en$\mathrm{Y}$ gastric bypass (RYGBP) is most commonly performed; the role of adjustable gastric banding (AGB) is declining; and gastric sleeve resection has been gaining importance $[7,8]$. One major complication of RYGBP is development of malabsorption of various nutrients, electrolytes, vitamins, and some drugs. Poor absorption of calcium can be of 
particular concern in patient subsets and may lead to secondary hyperparathyroidism [9-11].

One of the most common secondary complications of DM is renal failure, which commonly is associated with secondary hyperparathyroidism [12]. Although renal transplantation may reverse secondary hyperparathyroidism, commonly tertiary hyperparathyroidism has already developed and, therefore, removal of the parathyroid glands may be required. Most surgeons prefer 3.5 gland resection; however, also total parathyroidectomy with autotransplantation has been suggested [13]. Hypocalcemia is a common complication after parathyroidectomy and in severe cases is also referred to as hungry bone syndrome [14]; it seems to originate from a rapid drop in the PTH levels and may be more common in patients with secondary hyperparathyroidism. The profound hypocalcemia and hypophosphatemia may linger and require high-dose calcium and vitamin $\mathrm{D}$ supplementation. Administration of bisphosphonates has been suggested to prevent and/or treat the condition.

We herein report a 35-year-old woman with IDDM, who developed severe hypocalcemia after three-and-a-half-gland parathyroidectomy for tertiary hyperparathyroidism in the setting of being status post-RYGBP and SPK.

\section{Case Report}

A 35-year-old female was diagnosed with type I DM at the age of 9 years. During childhood her DM was poorly controlled and the patient gained significant weight. At the age of 25 years her weight was $105 \mathrm{~kg}$ with a body mass index (BMI) of $40 \mathrm{~kg} / \mathrm{m} 2$ and her renal function started to deteriorate with progression to requiring hemodialysis by age 30 . With development of renal failure, secondary hyperparathyroidism was noted. Due to her obesity, she was not eligible for a renal transplant or a SPK. At this point it was decided to offer her bariatric surgery, and, after extensive discussion, it was felt that a RYGBP was the best option for her in terms of weight loss. At the age of 32 years, she underwent uneventful roboticassisted surgery; the stomach remnant was attached to the abdominal wall for potential future access.

Over the next 2 years she lost $60 \mathrm{~kg}$ and underwent SPK during which the donor duodenal segment was diverted to a bowel loop distal to her Roux loop implant site into the common channel. Induction immunosuppression with alemtuzumab was followed by maintenance with tacrolimus (trough levels 5-7 ng/mL), mycophenolate-mofetil (2 g daily), and a steroid taper. She was CMV seronegative and received a graft from a CMV positive donor and received standard prophylaxis with oral ganciclovir (GCV) for 100 days. Within 100 days posttransplant, she was readmitted to the hospital with acute CMV disease, which was successfully treated with intravenous ganciclovir.

Shortly after this episode the patient was found to have skin lesions on her right leg, which were diagnosed as calciphylaxis. Her serum calcium at that time was $14 \mathrm{mg} / \mathrm{dl}$ and the diagnosis of tertiary hyperparathyroidism was made. A three-and-a-half-gland resection together with subtotal thymectomy was done without any complications; the left lower parathyroid gland was the only normal appearing and half of it was preserved taking care that blood supply remained intact. Intraoperative parathyroid hormone levels dropped from $>1500$ to 150 . Calcium serum levels within 24 hours postoperatively were $9 \mathrm{mg} / \mathrm{dl}$ with ionized calcium of $3.5 \mathrm{mg} / \mathrm{dl}$. She was discharged in good condition within 24 hours postoperatively with daily calcium supplementation of $4.5 \mathrm{~g} /$ day divided into three doses.

During the following week her calcium levels started to drop and on day 10 postoperatively at an outside hospital serum calcium was found to be critically low at $5.5 \mathrm{mg} / \mathrm{dl}$ with an ionized fraction of $2.1 \mathrm{mg} / \mathrm{dl}$. However, the patient had remained clinically symptom free. She was admitted for intravenous calcium replacement. Pushes of calcium were unable to appropriately raise her calcium levels and, therefore, a calcium drip $(85 \mathrm{mg} / \mathrm{h})$ was started. Her calcium levels came up to $7.1 \mathrm{mg} / \mathrm{dl}$. Oral calcium dose was raised to $15 \mathrm{~g} /$ day and hydrochlorothiazide was started. The calcium drip was stopped and the patient was discharged home in good condition.

Intense calcium supplementation was continued. Gradually the patient's gastrointestinal tract started to adapt and after two years her calcium levels started to stabilize. She has not experienced any additional complications from her transplant or gastric bypass. She is currently alive with excellent function of both grafts, normal calcium levels, stable weight, and an excellent quality of life almost five years after her last surgery.

\section{Discussion}

We report a patient with a complex medical history and multiple surgical interventions involving bariatric, transplant, and endocrine surgeons. The patient benefited from each of these interventions and all were clearly indicated. However, better communication between the various involved teams may have resulted in an even better outcome and prevention of the severe hypocalcemia following her three-and-a-halfgland parathyroidectomy. She was cured from her type 1 and type $2 \mathrm{DM}$ and since her successful SPK she is off any antidiabetic medication and is off dialysis and has regained an excellent quality of life having maintained a normal weight after bariatric surgery.

Development of obesity in patients with type I DM is rare and is associated with an excessive risk for secondary complications due to the superimposed peripheral insulin resistance [15]. Escalating amounts of exogenous insulin may be required in these patients in order to maintain acceptable blood glucose control. In combination with other comorbidities, development of secondary complications including renal failure is common such as in our patient. Once on dialysis, renal transplant was discussed but her obesity was considered a contraindication. Therefore, bariatric surgery was suggested and RYGBP seemed to be the most promising intervention to rapidly achieve the necessary weight loss.

Hypocalcemia is more common after RYGBP than after AGB or gastric sleeve resection as calcium absorption takes place mainly in the proximal gastrointestinal tract which is excluded in patients with RYGBP [16]. However, sleeve gastrectomy also may cause secondary metabolic complications 
with low calcium and elevated PTH levels [17]. Successful AGB has been reported in a SPK recipient who developed significant weight gain [18] and another SPK recipient underwent placement of a gastric pacemaker for gastroparesis associated with significant weight gain [19]. Limited data on sleeve gastrectomy in PTx are available but the procedure seems promising and appealing in this patient population [20-22]. Small series of patients with NIDDM undergoing PTx have been published; the treatment is reserved for patients who have lost significant weight and remain insulindependent [23].

In our patient, management of hypocalcemia was challenging [24]. Hungry bone syndrome may have been an important component leading to the very low serum calcium levels [14]. It is unclear if a RYGBP or a SPK transplant may exacerbate hungry bone syndrome. Even with $15 \mathrm{~g}$ of oral calcium and administration of vitamin $\mathrm{D}$, the patient had difficulties to maintain normal calcium levels due to limited calcium absorption. Vitamin D levels were kept above $50 \mathrm{ng} / \mathrm{ml}$ throughout the postoperative course. Palal et al. reported on life-threatening hypocalcemia after parathyroidectomy in a renal failure patient with previous RYGBP [24]. Hydrochlorothiazide increases calcium reabsorption and decreases renal calcium loss; however, use of diuretics in SPK recipients is controversial.

If the patient would not have been able to maintain adequate calcium levels in the long term, placing a gastrostomy tube into the stomach remnant under fluoroscopic guidance may have been an option. Calcium could be delivered directly into her proximal gastrointestinal tract, with improved absorption and resolution of hypocalcemia. Recently, recombinant parathyroid hormone has been introduced in clinical practice [25] for refractory cases of hypoparathyroidism but is reluctantly used in patients like ours. In fact PTH levels recovered in our patient and ultimately she was able to adjust to her unique metabolic challenge with highdose oral calcium and vitamin D administration. This complication of severe hypocalcemia after parathyroidectomy should be considered in patients with a history of RYGBP $[26,27]$.

\section{Conflicts of Interest}

The authors declare that they have no conflicts of interest.

\section{References}

[1] A. C. Gruessner and R. W. G. Gruessner, "Long-term outcome after pancreas transplantation: A registry analysis," Current Opinion in Organ Transplantation, vol. 21, no. 4, pp. 377-385, 2016.

[2] A. C. Gruessner and R. W. G. Gruessner, "Pancreas transplant outcomes for United States and non United States cases as reported to the United Network for Organ Sharing and the International Pancreas Transplant Registry as of December 2011," Clinical Transplantation, pp. 23-40, 2012.

[3] C. Fourtounas, "Transplant options for patients with type 2 diabetes and chronic kidney disease," World Journal of Transplantation, vol. 4, no. 2, pp. 102-110, 2014.
[4] A. C. Gruessner, M. R. Laftavi, O. Pankewycz, and R. W. G. Gruessner, "Simultaneous Pancreas and Kidney Transplantation-Is It a Treatment Option for Patients With Type 2 Diabetes Mellitus? An Analysis of the International Pancreas Transplant Registry," Current Diabetes Reports, vol. 17, no. 6, article no. 44, 2017.

[5] C. Margreiter, T. Resch, R. Oberhuber et al., "Combined pancreas-kidney transplantation for patients with end-stage nephropathy caused by type- 2 diabetes mellitus," Transplantation, vol. 95, no. 8, pp. 1030-1036, 2013.

[6] J. L. Colquitt, K. Pickett, E. Loveman, and G. K. Frampton, "Surgery for weight loss in adults," Cochrane Database of Systematic Reviews, vol. 8, Article ID CD003641, 2014.

[7] W. J. English, E. J. DeMaria, S. A. Brethauer, S. G. Mattar, R. J. Rosenthal, and J. M. Morton, "American Society for Metabolic and Bariatric Surgery estimation of metabolic and bariatric procedures performed in the United States in 2016," Surgery for Obesity and Related Diseases, vol. 14, no. 3, pp. 259-263, 2018.

[8] D. A. Hirth, E. L. Jones, K. B. Rothchild, B. C. Mitchell, and J. A. Schoen, "Laparoscopic sleeve gastrectomy: Long-term weight loss outcomes," Surgery for Obesity and Related Diseases, vol. 11, no. 5, pp. 1004-1007, 2015.

[9] J. Jin, A. V. Robinson, P. T. Hallowell, J. J. Jasper, T. A. Stellato, and S. M. Wilhem, "Increases in parathyroid hormone (PTH) after gastric bypass surgery appear to be of a secondary nature," Surgery, vol. 142, no. 6, pp. 914-920, 2007.

[10] P. Toelle, R. Peterli, I. Zobel, C. Noppen, C. Christoffel-Courtin, and T. Peters, "Risk factors for secondary hyperparathyroidism after bariatric surgery: a comparison of 4 different operations and of vitamin D-receptor-polymorphism," Experimental and Clinical Endocrinology \& Diabetes, vol. 120, no. 10, pp. 629-634, 2012.

[11] S. Hewitt, T. T. Søvik, E. T. Aasheim et al., "Secondary hyperparathyroidism, vitamin D sufficiency, and serum calcium 5 years after gastric bypass and duodenal switch," Obesity Surgery, vol. 23, no. 3, pp. 384-390, 2013.

[12] T. Amin, PT. Coates, J. Barbara, P. Hakendorf, and N. Karim, "Prevalence of Hypercalcaemia in a Renal Transplant Population: A Single Centre Study," International Journal of Nephrology, vol. 2016, Article ID 7126290, p. 2016, 2016.

[13] H. Gilat, R. Feinmesser, Y. Vinkler et al., "Clinical and operative management of persistent hyperparathyroidism after renal transplantation: A single-center experience," Head \& Neck, vol. 29, no. 11, pp. 996-1001, 2007.

[14] N. Jain and R. F. Reilly, "Hungry bone syndrome," Current Opinion in Nephrology and Hypertension, vol. 26, no. 4, pp. 250255, 2017.

[15] V. M. da Costa, P. de Carvalho Padilha, G. C. de Lima et al., "Overweight among children and adolescent with type I diabetes mellitus: prevalence and associated factors," Diabetology \& Metabolic Syndrome, vol. 8, no. 1, 2016.

[16] V. Newcombe, A. Blanch, G. H. Slater, A. Szold, and G. A. Fielding, "Laparoscopic adjustable gastric banding prior to renal transplantation," Obesity Surgery, vol. 15, no. 4, pp. 567570, 2005.

[17] N. Zarshenas, M. Nacher, K. W. Loi, and J. O. Jorgensen, "Investigating Nutritional Deficiencies in a Group of Patients 3 Years Post Laparoscopic Sleeve Gastrectomy," Obesity Surgery, vol. 26, no. 12, pp. 2936-2943, 2016.

[18] H. Bonatti, T. Schmitt, J. Northup et al., "Laparoscopic gastric banding in a kidney-pancreas transplant recipient with new 
onset type II diabetes mellitus associated with morbid obesity," Clinical Transplantation, vol. 22, no. 6, pp. 829-832, 2008.

[19] H. Bonatti, G. Brandacher, E. Hoeller et al., "Laparoscopically implanted gastric pacemaker after kidney-pancreas transplantation: Treatment of morbid obesity and diabetic gastroparesis," Obesity Surgery, vol. 17, no. 1, pp. 100-103, 2007.

[20] E. F. Elli, R. Gonzalez-Heredia, L. Sanchez-Johnsen, N. Patel, R. Garcia-Roca, and J. Oberholzer, "Sleeve gastrectomy surgery in obese patients post-organ transplantation," Surgery for Obesity and Related Diseases, vol. 12, no. 3, pp. 528-534, 2016.

[21] M. Porubsky, J. A. Powelson, D. J. Selzer et al., "Pancreas transplantation after bariatric surgery," Clinical Transplantation, vol. 26, no. 1, pp. E1-E6, 2012.

[22] J. Zelones, O. Biswas, and A. Mehran, "Laparoscopic sleeve gastrectomy after simultaneous pancreas-kidney transplant," The American Surgeon, vol. 78, no. 5, pp. 613-614, 2012.

[23] D. J. Lo, B. A. Sayed, and N. A. Turgeon, "Pancreas transplantation in unconventional recipients," Current Opinion in Organ Transplantation, vol. 21, no. 4, pp. 393-398, 2016.

[24] B. Palal, M. Sinsakul, and S. Reutrakul, "Life-Threatening Hypocalcemia following Subtotal Parathyroidectomy in a Patient with Renal Failure and Previous Roux-en-Y Gastric Bypass Surgery," Case Reports in Endocrinology, vol. 2011, Article ID 370583, p. 2011, 2011.

[25] G. Marcucci, G. Della Pepa, and M. L. Brandi, "Drug safety evaluation of parathyroid hormone for hypocalcemia in patients with hypoparathyroidism," Expert Opinion on Drug Safety, vol. 16, no. 5, pp. 617-625, 2017.

[26] S. M. Pietras and M. F. Holick, "Refractory hypocalcemia following near-total thyroidectomy in a patient with a prior roux-en-y gastric bypass," Obesity Surgery, vol. 19, no. 4, pp. 524526, 2009.

[27] J. A. Gross, S. M. Olsen, C. A. Koch, and E. J. Moore, "Severe symptomatic hypocalcemia following total thyroidectomy in a patient with a history of Roux-en-Y gastric bypass surgery," Ear, Nose \& Throat Journal, vol. 93, no. 1, p. E6, 2014. 


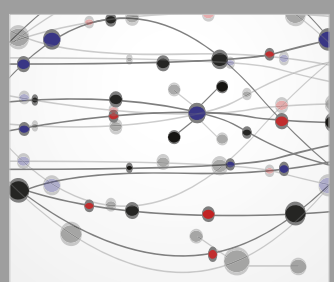

The Scientific World Journal
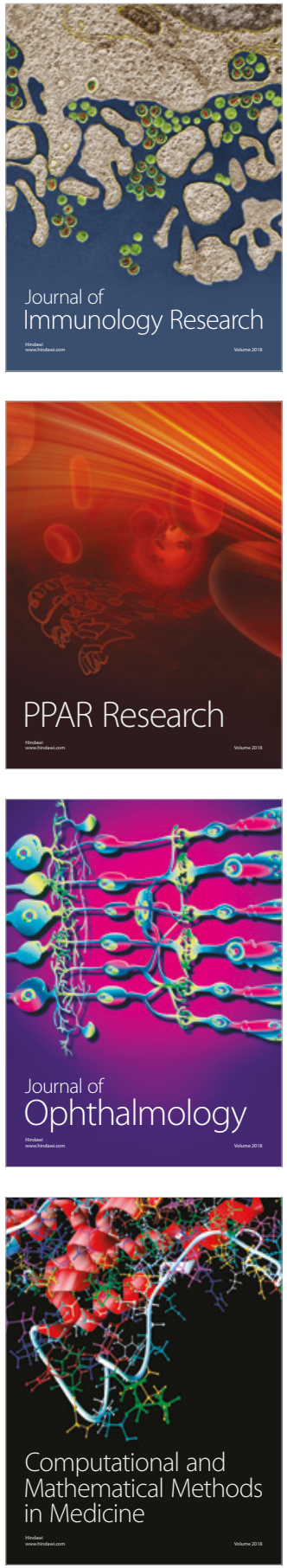

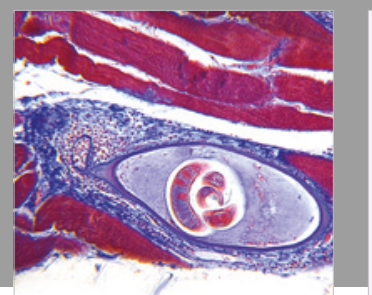

Gastroenterology Research and Practice

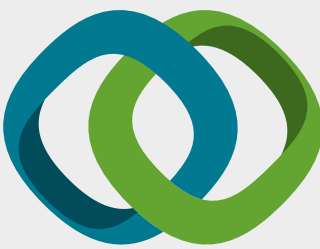

\section{Hindawi}

Submit your manuscripts at

www.hindawi.com
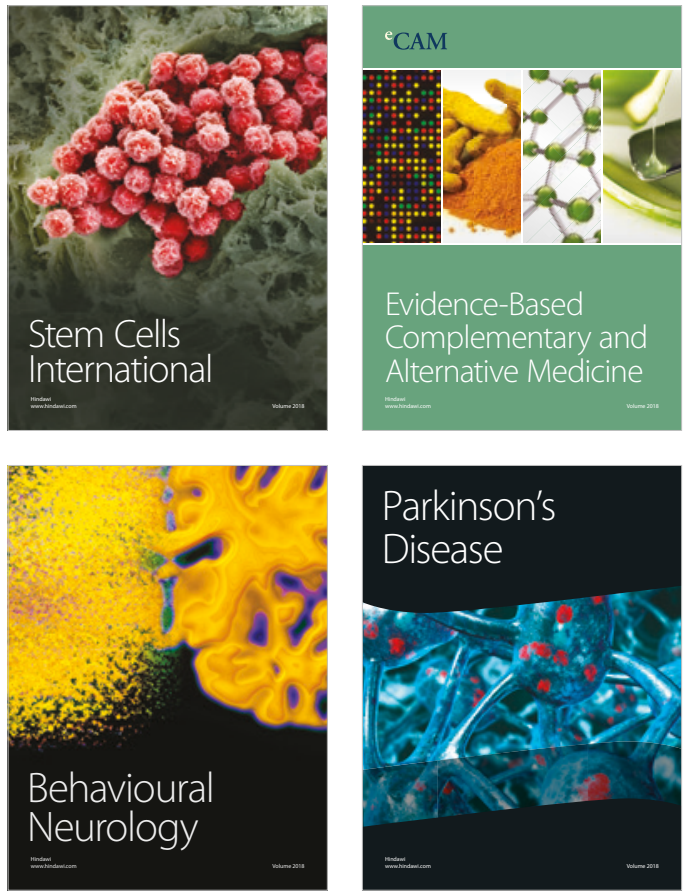

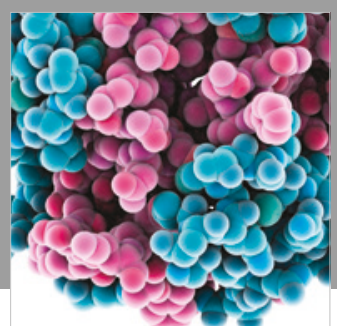

ournal of

Diabetes Research

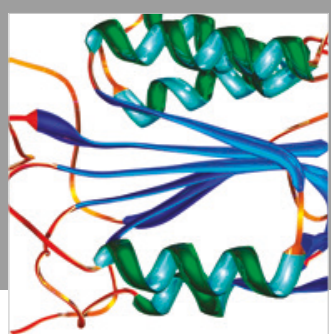

Disease Markers
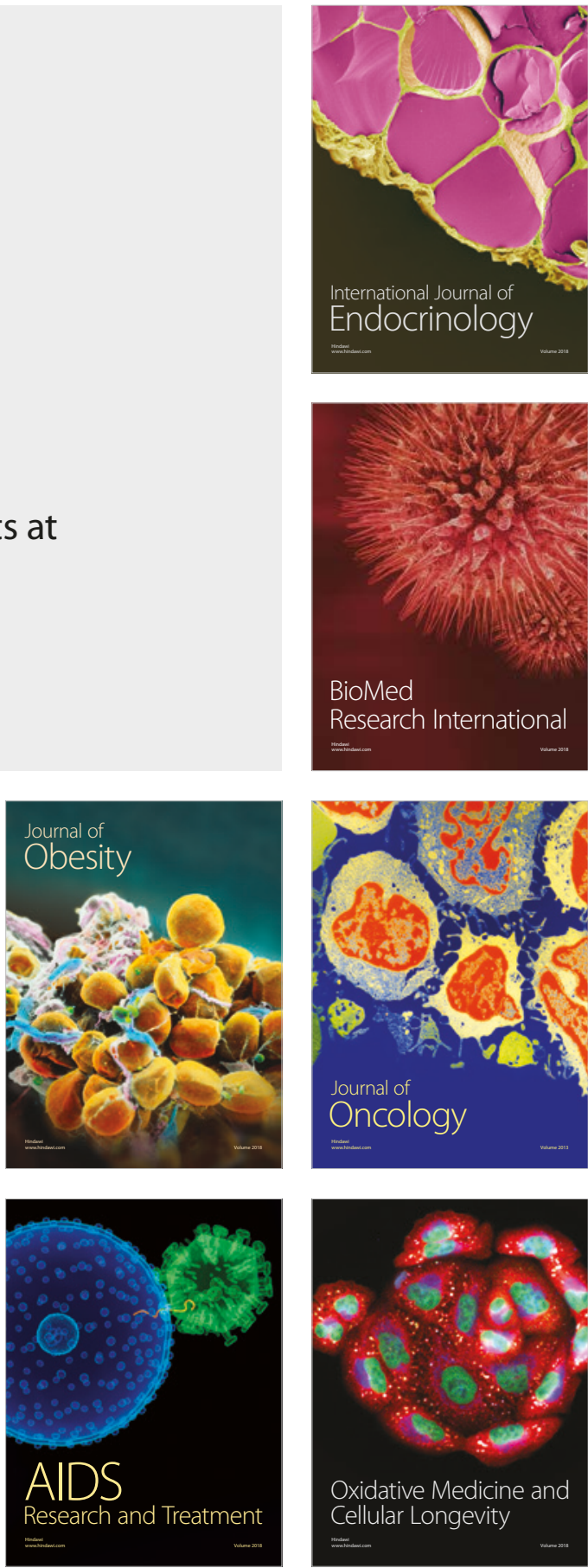